\title{
Measurement and Analysis of the Field Quality of LHC Prototype and Pre-Series Superconducting Dipoles
}

\author{
M. Aleksa, S. Amet, Z. Ang, L. Bottura, M. Buzio, P. Ferracin, O. Pagano, V. Remondino, S. Russenschuck, \\ S. Sanfilippo, W. Scandale, and E. Todesco
}

\begin{abstract}
We report the main results of the magnetic field measurements performed on the full-size LHC superconducting dipoles tested at CERN since summer 1998. Main field strength and field errors are summarized. We discuss in detail the contributions related to the geometry of the collared coil, the assembled cold mass, cool-down effects, magnetization of the superconducting cable and saturation effects at high field. Dynamic effects on field harmonics, such as the field decay during injection and field errors during current ramps, are assessed statistically.
\end{abstract}

Index Terms-Accelerator magnets, field quality, magnetic measurements.

\section{INTRODUCTION}

$\mathbf{T}$ HE MAGNETS for the LHC must satisfy strict requirements regarding field quality. Harmonic errors in the superconducting magnets will affect important beam parameters such as orbit, tune, coupling and chromaticity. The requirements have been expressed as a set of maximum field imperfections to be reached at operation [1]. A comparison of these requirements to the field homogeneity realistically achievable during series production of dipole magnets shows that active correction is necessary [2]. The correction system however can be efficient only if the field errors are known at operating conditions. This calls for systematic testing of dipoles in cold conditions and for a rigorous analysis of the correlation between the measured harmonics and the components of the cold mass, e.g., coil, collars and iron, critical for field quality.

As in warm electromagnets, in superconducting magnets two important contributions to the field errors are generated by the geometry of the coils and the saturation of the iron yoke. In addition, three other contributions to the errors stem from the superconducting properties of the cable and are especially important at low fields: persistent currents in superconducting filaments, eddy currents in the strands and in the cable during ramps and field decay and snap back at the change from constant current operation to the beginning of the ramp.

We have used this as a frame to organize the results of field quality aspects in the long superconducting LHC

Manuscript received September 24, 2001.

M. Aleksa, S. Amet, L. Bottura, M. Buzio, P. Ferracin, O. Pagano, V. Remondino, S. Russenschuck, S. Sanfilippo, W. Scandale, and E. Todesco are with CERN, CH-1211 Geneva 23, Switzerland (e-mail: stephane.sanfilippo@cern.ch).

Z. Ang is with TRIUMPH, Vancouver, BC, Canada.

Publisher Item Identifier S 1051-8223(02)03468-1. dipoles with austenitic steel collars tested so far. These are the 15-m long prototypes MBP2N2, MBP2O1, MBP2O2 and MBP2A2 and the first three of the 90 pre-series dipoles, HCLBB_001-CR001001, HCLBB_001-CR001 001 and HCLBB_001-CR002001, referred to as MBPSO01, MBPSO02 and MBPSA01 respectively. A detailed description of the design, of the main parameters and of the main assembly variants can be found in [3] for the prototypes and in [4] for the pre-series dipoles. The systems for field measurement in warm and cold conditions are described elsewhere [5], [6].

\section{MAIN FIELD}

\section{A. Transfer Function}

The dipole transfer function measured in the straight part of the coil is plotted in Fig. 1 for selected prototypes and for the pre-series dipoles. Experimental data are compared to calculations performed taking into account the different magnetic yoke geometry in prototypes and pre-series dipoles and in particular the shape of the ferromagnetic inserts between yoke and collars [2]. For a given current, the transfer function varies for the prototypes and pre-series dipoles in relative range of $6 \times 10^{-4}$. The calculated values are in agreement within the same range. This is at the limit of the beam dynamics requirements $(1 \sigma=$ $3 \times 10^{-4}$ ). More statistics are needed to confirm this value for the pre-series. The observed spread may be due to non nominal pole shims used during collaring.

\section{B. Magnetic Length}

The nominal magnetic length of the LHC dipoles is $14.3 \mathrm{~m}$. All dipoles measured (with an accuracy of $10^{-4}$ ) exceeded this value (see Fig. 2), apart from the prototype MBP2O2 that has a magnetic length $0.2 \%$ smaller than the other dipoles. The reasons are related to two assembly parameter differences. $\mathrm{MBP} 2 \mathrm{O} 2$ is the first $15-\mathrm{m}$-long dipole having a longitudinal symmetric yoke with a reduced length (the other prototypes had a longer asymmetric yoke) and is the last dipole equipped with non magnetic laminations in the ends of the magnet. On the other hand the pre-series dipoles have nested laminations, i.e., non magnetic laminations with reduced diameter complemented by an outer ferromagnetic ring. These two differences result in a reduction of the end contribution to the integrated field and thus in a shorter magnetic length of $\mathrm{MBP} 2 \mathrm{O} 2$ with respect either to the other previous prototypes or to the following 


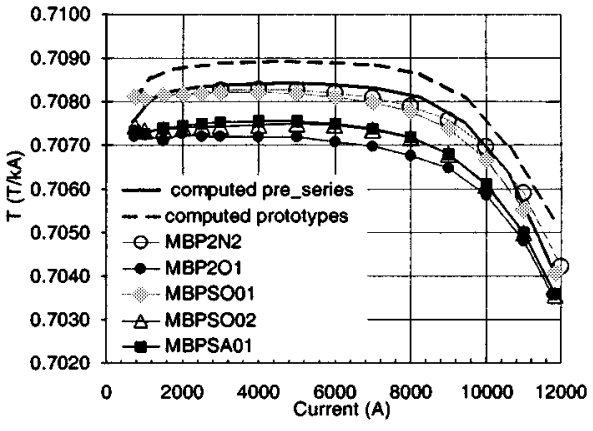

Fig. 1. Transfer functions measured in the prototypes and the pre-series dipoles compared to calculations. The different yoke geometry of prototype and pre-series dipoles was taken into account for the calculations.

pre-series. The difference in length among the two apertures of a dipole $(\Delta l$ in Fig. 2$)$ is at most $3 \mathrm{~mm}\left(2 \times 10^{-4}\right)$. Note that in most cases, the first aperture has systematically a higher magnetic length than the second one. Finally for the pre-series dipoles, the standard deviation of the relative spread over the six measured apertures $2.7 \times 10^{-4}$, is in agreement with the tolerance of $3 \times 10^{-4}$ given by beam dynamics [1].

\section{GEOMETRIC ERRORS}

\section{A. Warm-Cold Correlation}

Warm measurements (i.e., at $300 \mathrm{~K}$ ) can give a good evaluation of the geometric field errors. This is demonstrated in Fig. 3 where we have plotted the correlation between the loworder allowed harmonics measured in warm conditions (completed cold mass) and the geometric value in cold conditions, both averaged in the straight part of the coil. Warm measurements were performed at 12 A current with positive and negative current to eliminate the residual magnetization. The geometric harmonics at cold have been computed as the average of the measured values on the ramp up and ramp down powering branches at $5 \mathrm{kA}$. The correlation with the slope one is very good and offsets small. Table I reports the standard deviation of the warm-cold correlation $\sigma_{\text {warm-cold }}$ for normal and skew low-order harmonics up to the decapole. The standard deviation defined as in [7], corresponds to the spread around the ideal correlation line. It provides an estimate of the range of field quality control that can be achieved during series production in the case that warm measurements only are used to provide feed back on the coil geometry. $\sigma_{\text {warm-cold }}$ is about three times lower than the natural spread of the harmonics from magnet to magnet [2]. This confirms our expectation that measurements at $300 \mathrm{~K}$ are an effective means to control the geometric harmonics of a dipole in operating conditions. The small offset $\Delta_{\text {offset }}$ observed could be interpreted as an effect of the different thermal contractions of the dipole components after cool-down.

\section{B. Coil Size and Azimuthal Shims}

The coil used for the prototypes and the first pre-series dipoles do not have the same azimuthal size because of small variations of cable size or insulation thickness. In addition the collar dimensions were adjusted in the final iteration toward pre-series

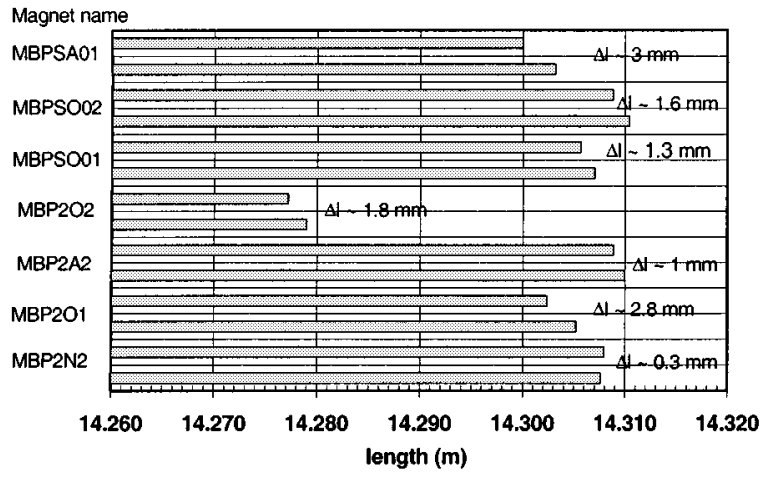

Fig. 2. Magnetic length at nominal current for the prototypes and the pre-series dipoles. The nominal magnetic length is $14.3 \mathrm{~m}$. Each bar describes the length of one aperture (two lengths per magnet).
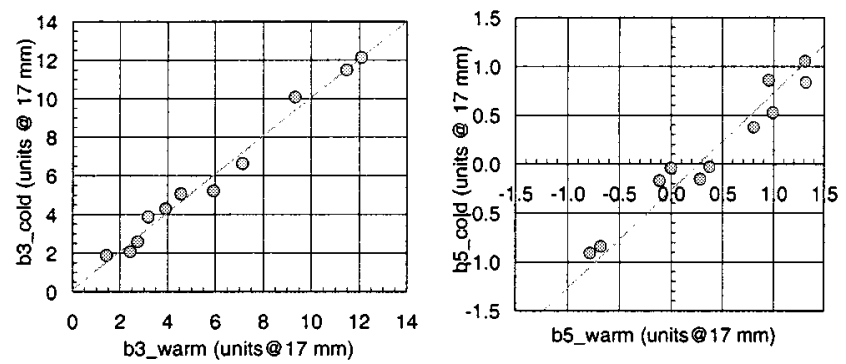

Fig. 3. Scatter plot of the normal sextupole (left) and decapole (right) measured in warm and cold conditions (geometric part only). Experimental points are compared with a linear fit with a slope 1 for the ideal correlation.

TABLE I

STANDARD DEVIATION AND OfFSET OF THE CORRELATION BETWEEN MEASUREMENTS OF GEOMETRIC HARMONICS AT $300 \mathrm{~K}$ AND AT 1.9 K (IN UNITS AT $17 \mathrm{~mm}$ )

\begin{tabular}{ccccc}
\hline $\begin{array}{c}\text { Order } \\
\mathrm{n}\end{array}$ & $\begin{array}{c}\sigma_{\text {warm-cold }} \\
\mathrm{b}_{\mathrm{n}}\end{array}$ & $\begin{array}{c}\Delta_{\text {offset }} \\
\mathrm{b}_{\mathrm{n}}\end{array}$ & $\begin{array}{c}\sigma_{\text {warm-cold }} \\
\mathrm{a}_{\mathrm{n}}\end{array}$ & $\begin{array}{c}\Delta_{\text {offset }} \\
\mathrm{a}_{\mathrm{n}}\end{array}$ \\
\hline 2 & 0.15 & -1.08 & 0.08 & 0.05 \\
3 & 0.49 & 0.07 & 0.06 & -0.08 \\
4 & 0.02 & -0.03 & 0.06 & 0.00 \\
5 & 0.17 & -0.27 & 0.03 & 0.00 \\
\hline
\end{tabular}

production. To compensate for the dimensional errors, supplementary pole shims were added between the coils and the collars. The difference $\delta$ with respect to the nominal pole shim was at most $0.3 \mathrm{~mm}$ for the first pre-series MBPSO01. The influence of the azimuthal coil size on the allowed harmonics was analyzed, compensating the collar deformation using the method described in [7]. The variation of the azimuthal size is computed for each pole and layer from the additional, known shim thickness $\delta$ reduced by $10 \%$.

Fig. 4 reports the correlation between the harmonic variation reconstructed using the above procedure and the geometric harmonics measured in cold conditions. The shape of the collar was modified from prototype to pre-series dipoles to reduce the effect of collar deformation [8], hence the prototype and pre-series magnets should be considered as two separate families. The data of each family were fitted with a line of fixed slope one. In Table II we report the value of the offset $\Delta_{\text {offset }}$ and the spread around the correlation line $\sigma_{\text {comp }}$. As shown in the Fig. 4 , the effect of a variation of the azimuthal coil size is in expected direction and magnitude. A rather larger spread is observed (see 
Table II) but it is compatible with the constraints given by beam dynamics [1]. The prototypes and the pre-series dipoles do not show an evident difference in this respect. The spread can be due to variation in coil geometry, assembly procedures and manufacturers. The presence of an offset reflects a non zero value of odd multipoles foreseen in the nominal design. The difference between this offset and the nominal value is mainly due to deformation of the collars during the assembly, not taken into account in the reconstruction. A complete analysis of the different behaviors between prototypes, pre-series and nominal design is given in [9].

\section{SATURATion AT High Field}

\section{A. Transfer Function}

As shown in Fig. 1, the saturation of the ferromagnetic yoke results in a reduction of the transfer function at nominal field by approximately 56 units with respect to the geometric value. This reduction is in good agreement with the prediction. Once the measured values are shifted vertically to the match the calculation at $5 \mathrm{kA}$, the error between calculation and measurement at nominal field is at most 6 units.

\section{B. Higher Order Harmonics}

In Fig. 5, we display the measured high field behavior of quadrupole $\left(b_{2}\right)$, sextupole $\left(b_{3}\right)$, octupole $\left(b_{4}\right)$ and decapole $\left(b_{5}\right)$ in the pre-series dipoles as compared to the variation expected based on the saturation of the ferromagnetic yoke [10]. The measured values have been shifted vertically to match the computed value at $5 \mathrm{kA}$, thus removing the effect of different geometry among the magnets. The calculation is in satisfactory agreement with the measurements for even multipoles. For $b_{3}$ and $b_{5}$, a significant discrepancy is visible at high current. In particular for $b_{5}$ the measured average high field variation is about 0.15 units, while a negligible variation is expected. We have found that the difference between the measured and computed values varies linearly with the square of the current. This indicates that the high field variation of the allowed harmonics may be caused by elastic compression of the coil under the electromagnetic loads.

\section{Contributions of the Superconducting CABle}

\section{A. Persistent Currents}

The magnetization associated with persistent currents in the $\mathrm{NbTi}$ superconducting filaments affects the allowed harmonics, especially at low fields. This contribution is therefore important at injection field $(0.54 \mathrm{~T})$. Fig. 6 summarizes the values of the field contribution from persistent currents measured at injection in the prototypes and in the pre-series dipoles. The persistent current effect is computed as the difference between the harmonic value at injection current and its geometric value. For comparison, the upper and lower $1 \sigma$ bounds of the expected contribution are reported in the same graph.

The order of magnitude measured matches roughly the expectation, except for MBPSO02 that displays an unusually smaller contribution $(-16 \%)$
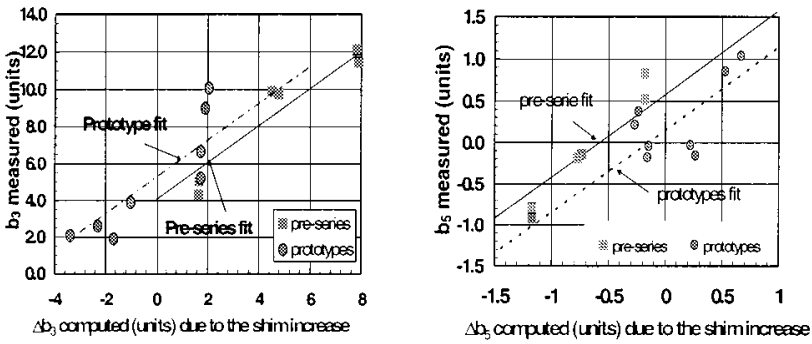

Fig. 4. Scatter plot of the variation of the geometric normal sextupole and decapole (ends excluded), as measured and reconstructed by calculation (taking into account as build geometry).

TABLE II

OfFSET AND Standard DeViation of the CorRelation Between GEOMETRIC HARMONICS AND RECONSTRUCTION (IN UNIT AT 17 mm)

\begin{tabular}{ccccc}
\hline $\begin{array}{c}\text { Harmonic } \\
\text { order }\end{array}$ & $\begin{array}{c}\text { Prototypes } \\
\Delta_{\text {offset }}\end{array}$ & $\sigma_{\text {comp }}$ & $\begin{array}{c}\text { Pre-series } \\
\Delta_{\text {offset }}\end{array}$ & $\sigma_{\text {comp }}$ \\
\hline $\mathrm{b}_{3}$ & 5.25 & 1.51 & 4.03 & 1.04 \\
\hline $\mathrm{b}_{\mathrm{s}}$ & 0.15 & 0.52 & 0.58 & 0.27 \\
\hline
\end{tabular}
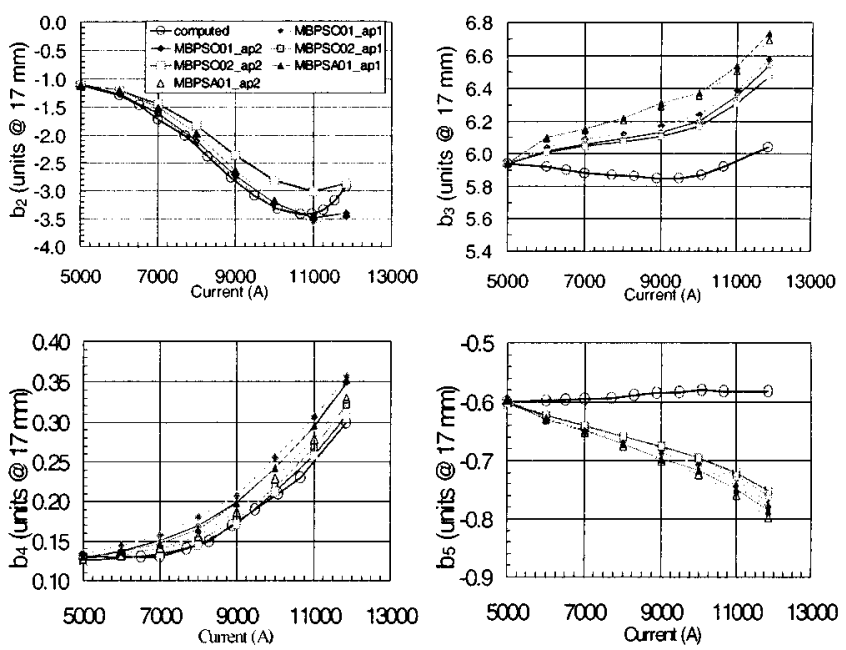

Fig. 5. Variation of the normal quadrupole, sextupole, octupole and decapole as a function of the current and comparison to the computed values.

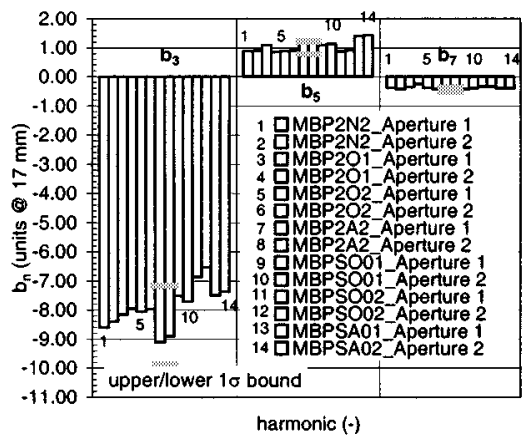

Fig. 6. Contribution of the persistent current to the field errors for the prototypes and the pre-series dipoles. The measured values are compared to the expected ones (upper and lower $1 \sigma$ bound). The different apertures of the dipoles are numbered from 1 to 14 to help the understanding.

\section{B. Eddy Currents}

During the acceleration of the injected particles, the dipole magnet is ramped at a maximum ramp-rate of $10 \mathrm{~A} / \mathrm{s}$, cor- 


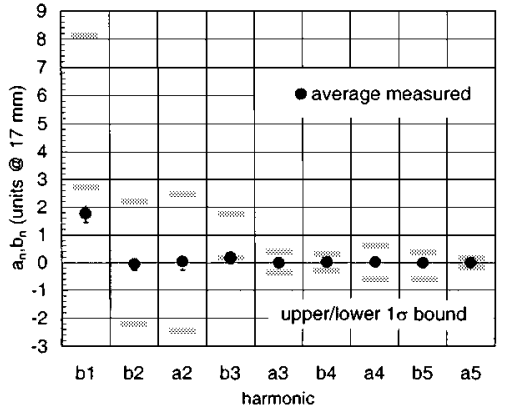

Fig. 7. Contribution of the coupling currents to the field errors for the prototypes and the pre-series dipoles. The measured values are compared to the expected ones (upper and lower $1 \sigma$ bound).

responding to a field change rate of approximately $7 \mathrm{mT} / \mathrm{s}$. Coupling currents in the strands and cables induce field errors proportional to the ramp-rate. The main contribution originates from cable coupling currents closing through the interstrand resistance $R_{c}$. The value of $R_{c}$ is controlled during cable production and is expected to be above $20 \mu \Omega$. Fig. 7 shows the average contribution of coupling currents measured in the prototypes and pre-series dipoles, compared to the expected values for a $R_{c}$ of $20 \mu \Omega$. The measured values are referred to a ramp starting at injection with the maximum rate of $10 \mathrm{~A} / \mathrm{s}$. As shown in the Fig. 7, this contribution is small for all harmonics and has significantly lower values than the expected ones. This implies that the average $R_{c}$ is well above $20 \mu \Omega$ in the dipoles tested so far [11].

\section{Decay and Snapback}

Superconducting dipoles are characterized by a significant drift of the magnetic field (decay) when the current is constant with typical time scales in the order of several minutes to several hours [12], [13]. The decay contribution to the field errors, measured over a simulated injection plateau of $1000 \mathrm{~s}$, is summarized in Fig. 8. Only the envelope of the values measured on all magnets tested so far has been plotted.

The same pre-cycling conditions have been used for all measurements, starting with a quench and a current cycle with a flat top of $30 \mathrm{~min}$. at the nominal current of $11850 \mathrm{~A}(8.4 \mathrm{~T})$. The maximum decay expected is estimated as roughly one third of the persistent current contribution to the field. In Fig. 8, the upper and lower expected $\sigma$ bound are also reported. The measurements are in accordance with these upper and lower bound estimates. In particular for the normal sextupole, the upper limit appears conservative and for the decapole the $\sigma$ is large.

\section{CONCLUSION}

Our understanding of the field quality in the 15-m long LHC dipoles progresses steadily as more magnets are being produced. We are presently confident that the geometric harmonics measured at warm are well correlated to the values under oper-

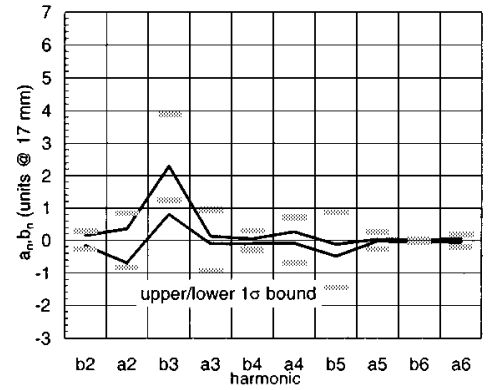

Fig. 8. Expected ( $1 \sigma$ bound) and measured decay contribution to the field errors in the prototypes and in the pre-series dipoles. The two solid lines represent the envelope of the measured values.

ating conditions. Fine control of the geometric harmonics can be achieved acting on assembly features such as pole shims. Similarly the yoke saturation effect appears to be broadly understood, although the measurements at high field indicate an elastic deformation of the coil originally not foreseen in the field errors budget. Among the contributions related to superconducting effects, the field errors generated by persistent currents are the strongest and show the largest absolute variation from magnet to magnet. This contribution will therefore require attention and careful characterization. At present both the field errors caused by coupling currents in the cables and the field decay at injection are smaller than initially allocated in the error budget.

\section{REFERENCES}

[1] O. Brüning and S. Fartoukh, "Field quality specifications for the LHC main dipole magnets,", LHC Project Report, 2001, to be published.

[2] P. Ferracin et al., "Control of the field quality for the production of the main LHC dipole,", LHC Project Report 467, 2001.

[3] M. Modena et al., "Manufacture and performance of the LHC main dipole final prototypes," in Proc. 2000 Part. Acc. Conf., Vienna, Austria, 2000, pp. 2144-2148.

[4] M. Modena et al., "Final prototypes, first pre-series units and steps toward series production of the main LHC dipoles," in Proc. IEEE Part. Acc. Conf., Chicago, IL, USA, 2001.

[5] J. Billan et al., "Ambient temperature field measuring system for LHC superconducting dipoles," Proc. IEEE Trans. Magn., vol. 32, pp. 3073-3076, 1996.

[6] J. Billan et al., "Twin rotating coils for cold magnetic measurements of 15-m long LHC dipoles," IEEE Trans. Appl. Superconduct., vol. 10, pp. 1422-1426, Mar. 2000.

[7] Z. Ang et al., "Coil size and geometric field quality in short model dipoles for LHC," Proc. IEEE Trans. Appl. Superconduct., vol. 10, pp. 53-56, Mar. 2000.

[8] D. Perini, CERN, LHC Division, CH-1211 Geneva 23, Switzerland, personal communication.

[9] P. Ferracin et al., "Impact of coil deformations on field quality in the large hadron collider main dipole,", submitted for publication.

[10] S. Russenschuck, 1st Int. Roxie Users Meeting and Workshop, S. Russenschuck, Ed. Geneva, Switzerland: CERN, 1999.

[11] A. Akmethov et al., "Loss and dynamic magnetic field measurements in LHC dipoles,", submitted for publication.

[12] M. Haverkamp et al., "Studies of decay and snapback effects on LHC dipole magnets," in EUCAS'99, Barcelona, Spain, 1999, Proc. Inst. Phys. Conf. Ser. No 167.

[13] - "Studies of decay and snapback effects on LHC dipole magnets," in EUCAS'99: IOP, 2000, pp. 1183-1186. 